\title{
Influencia de las concentraciones en iones hidróxilos, carbonatos y cloruros sobre la corrosión por picaduras del acero en el hormigón
}

\author{
Influence of hydroxyls, carbonates and chlorides \\ ions on the pitting corrosion of steel in concrete
}

THIERRY CHAUSSADENT Y ROGER DRON

Laboratoire Central des Ponts et Chaussées, 58 Boulevard Lefebvre 75732 París cedex, 15, FRANCE

\section{RESUMEN}

Los procesos electroquímicos de corrosión de las armaduras de acero del hormigón son estudiados simulando su fase líquida mediante disoluciones sintéticas que cubren playas importantes en iones hidróxilos, carbonatos y cloruros. El acero está sometido o no a una polarización eléctrica.

Este análisis ha permitido definir un nuevo parámetro significativo, la relación [Cr]/[AIK] (siendo AlK la alcalinidad total), que caracteriza las condiciones de corrosión por picaduras del acero polarizado.

Además, el estudio ha mostrado que una polarización, a un potencial más bajo que - $700 \mathrm{mV}$ scE impide los mecanismos de corrosión.

\section{$S U M M A R Y$}

The electrochemical process of steel corrosion in concrete are studied by simulating its liquid phase with synthetical solutions in a large range of hydroxyls, carbonates and chlorides ions. The steel specimen is either under or without an electrical polarization.

The present analysis has made it possible to define a new relevant parameter, which is the [CI]/[AIK] ratio (where AlK is the total alkalinity). It characterizes the conditions of the polarized steel pitting corrosion.

Furthermore, the investigation showed out that a polarization at potentials lower than $-700 \mathrm{mV} V_{\text {sCE }}$, stops the ccrrosion processes.

\section{INTRODUCCIÓN}

La alcalinidad de la solución en los poros del hormigón confiere generalmente una buena protección a las armaduras de acero contra la corrosión. Esta alcalinidad está ligada a la portlandita formada durante la hidratación del cemento, y, después de algunos meses, al incremento de las concentraciones en iones alcalinos (hasta $0,6 \mathrm{M}$ ).

Sin embargo, ciertas circunstancias pueden alterar esta pasivación, e inclusive acarrear la destrucción mecánica del hormigón debida a la formación de voluminosos productos de corrosión. La carbonatación del hormigón, por su influencia sobre la basicidad, así como la presencia de iones cloruros, son los dos principales factores capaces de conducir a dicha situación, sobre todo en presencia de oxígeno.

\section{INTRODUCTION}

The high $\mathrm{pH}$ value of the pore liquid in concrete results generally in a good protection of reinforcing steels against corrosion, This alkalinity is related with the portlandite formed during the cement hydration, then, after few months, with the increase of alkaline ions contents (up to $0.6 \mathrm{M}$ ).

Nevertheless, some conditions can deteriorate this passivation, induce the corrosion process and even result in the mechanical failure of the concrete by forming voluminous corrosion products. The concrete carbonation, with its effect on the $\mathrm{pH}$ value, and the presence of chloride ions are the two main factors which can lead to this condition, mainly when oxygen is present. 
Numerosos autores (1-5) han estudiado la corrosión de las armaduras de acero en el hormigón utilizando disoluciones sintéticas. Este estudio tiene por objeto precisar la influencia de las especies químicas contenidas en dichas disoluciones. Por esta razón son estudiadas ciertas disoluciones cuya composición está alejada de la del agua en los poros del hormigón.

Esta investigación fue llevada a cabo con la ayuda de ensayos potenciocinéticos con la finalidad de mejorar estos conocimientos y también para definir las condiciones de corrosión (por picaduras) y de protección.

\section{PROCEDIMIENTOS EXPERIMENTALES}

\section{Especimen de acero y disoluciones estudiadas}

Los especímenes metálicos son redondos (diámetro $6 \mathrm{~mm}$ ) en acero al carbono (Fe E 235) corrientemente utilizado en las obras de hormigón armado. Son embebidos en una resina y la superficie ensayada, perpendicular al eje del cilindro, es pulida mecánicamente (papel abrasivo $600)$ y desengrasada con acetona antes de cada ensayo.

Las soluciones estudiadas fueron preparadas a base de agua destilada (hervida para eliminar el gas carbónico disuelto) y de productos químicos "puros para análisis", que son $\mathrm{NaOH}, \mathrm{KOH}$, $\mathrm{NaHCO}_{3}$ y $\mathrm{Na}_{2} \mathrm{CO}_{3}$. Se debe observar que estas disoluciones solamente representan parcialmente la naturaleza química del hormigón, debido a la ausencia de iones calcio. Por esta razón ha sido posible cubrir playas importantes de concentraciones en iones cloruros, carbonatos e hidróxilos. En esta investigación, la primera serie de ensayos fue efectuada en disoluciones de sosa o de potasa, la segunda en disoluciones carbonatadas.

\section{Aparato de medida y técnica de explotación}

Los ensayos electroquímicos, fundados en el trazado de las curvas potenciocinéticas y la medida de los potenciales de corrosión al abandono son completados por la caracterización de los productos de corrosión, al abandono son completados por la caracterización de los productos de corrosión, intermediarios y finales.

Para los ensayos potenciocinéticos, se utilizó un aparato compuesto de un potenciostato Tacussel tipo PRT 20-2X equipado con un piloto Servovit, y una célula electroquímica de ensayo convencional con tres electrodos. El electrodo de referencia es de calomelanos saturado en $\mathrm{KCl}$. Este dispositivo experimental, presentado en la figura 1, permite hacer variar el potencial del metal desde una
Many authors (1-5) have studied the corrosion of reinforcing steel in concrete by using synthetical solutions. The object of the present investigation is to precise the influence of the chemical species contained in these solutions. Therefore, it deals with solutions some of which are very far from the the real pore solution in concrete.

This investigation was carried out by using potentiodynamic tests, in order to improve the knowledge, and also to determine the conditions of steeel corrosion (pitting) and of protection.

\section{EXPERIMENTAL}

\section{Steel and solutions studied}

The metallic specimens were cylinders (6 $\mathrm{mm}$ diameter) made of carbon steel (French grade $F e E 235)$ commonly used in reinforced concrete structures. They were coated with resins and the test area was a cross section which was mechanically polished (paper grade 600) and degreased with aceton before any testing.

The solutions studied were prepared by using distilled water (boiled for eliminating the carbon dioxyde dissolved) and chemical products "pure for analyses", which were $\mathrm{NaOH}, \mathrm{KOH}, \mathrm{NaCl}$, $\mathrm{NaHCO}_{3}$ and $\mathrm{Na}_{2} \mathrm{CO}_{3}$. It is to be noticed that these solutions represent only partially the real nature of concrete, because they contain no calcium ions. But they make it possible to study the effect of large ranges of chlorides, carbonates and hydroxyls ions contents. For the present investigation, the first series of tests was carried out with $\mathrm{NaOH}$ and $\mathrm{KOH}$ solutions. The second series dealt with carbonated solutions.

\section{Experimental arrangement and technique}

The electrochemical tests, including potentiodynamic test plotting and zero current potential readings were completed with the characterization of intermediate and final products.

For the potendiodynamic tests, the arrangement included a potentiostat Tacussel PRT 20-2X type, with a Servovit pilot and a conventional three electrodes test cell. The reference electrode was saturated calomel electrode with KCL. With this experimental arrangement (figure 1), the metal potential could change, first from a value corresponding to immunity (- $1.100 \mathrm{mV}$ SCE $)$ up to 


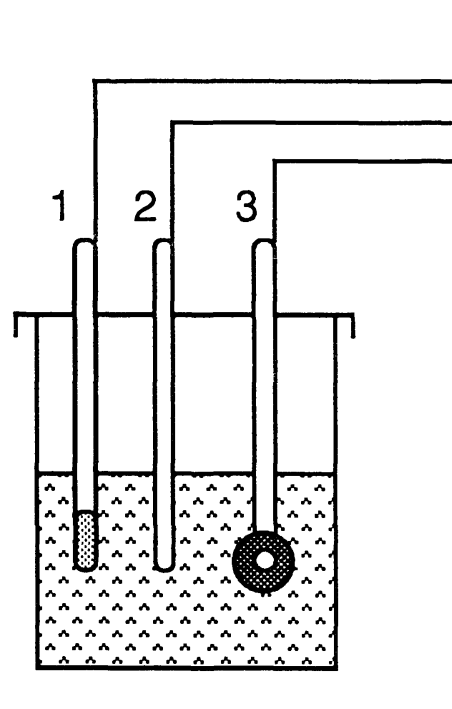

Potenciostato

registrador.

(Pilot

potentiostat

recorder).

(1) Contra electrodo $(P t)$. Counter electrode $(P t)$.

(2) Electrodo de referencia $(\mathrm{Hg} / \mathrm{HgCl})$. Reference electrode $(\mathrm{Hg} / \mathrm{HgCl})$.

(3) Electrodo de trabajo (acero). Work electrode (steel).

Fig. 1.-Celda de corrosión.

Fig. 1.-Corrosion cell.

posición que corresponde a la inmunidad $\left(-1.100 \mathrm{mV}_{\mathrm{SCE}}\right)$ hasta valores para los cuales la corrosión se vuelve intensa (la densidad de corriente alcanzado hasta $100 \mathrm{~mA} . \mathrm{cm}^{-2}$ ) y en seguida volver al dominio de protección.

La velocidad de barrido en estos ensayos fue de $5 \mathrm{mV}$. $\mathrm{min}^{-1}$. Los ensayos fueron efectuados en una célula hermética sin ningún barboteo de gas, a temperatura ambiente y sin agitación. La mayor parte de ellos fueron duplicados.

Siendo la célula en vidrio transparente, los productos formados en la superficie del acero son visibles. Además, al final del ensayo potenciocinético, el estado del acero fue examinado visualmente y ciertos productos fueron identificados por difracción de rayos $X$.

Las curvas potenciocinéticas obtenidas, como la esquematizada en la figura 2, tienen cuatro dominios característicos:

1) un dominio de inmunidad (con

desprendimiento de hidrógeno),

2) un dominio de pasivación: esta zona de potenciales, más o menos extendida, está delimitada, de una parte, por varios picos de endeble intensidad, que caracterizan los equilibrios hierros/hidróxidos, hidróxidos/óxidos $(6-8)$ y de otra parte, por un potencial $\mathrm{Er}$ (potencial de ruptura a partir del cual las picaduras aparecen),

3) un dominio de propagación de dichas picaduras,

4) un dominio correspondiente a la curva de entorno hacia potenciales más negativos; esta parte conduce a un potencial Ep (llamado potencial de protección o de fin de propagación de las picaduras). values for which the corrosion was high (current density about $100 \mathrm{~mA} . \mathrm{cm}^{-2}$ ), then back down to the passivation range.

The sweep rate was equal to $5 \mathrm{mV} \cdot \mathrm{mn}^{-1}$ for all the tests. These tests were carried out an airtight cell, without any gas introduction, at the ambient temperature $\left(20^{\circ} \mathrm{C}\right)$ and without any agitation. Most of the potentiodynamic tests were duplicated.

The cell was of transparent glass and the products formed on steel surface were visible. Moreover, at the end of a potentiodynamic test, the steeel suface state was examined and some corrosion products were identified by $X$-ray diffraction.

The potentiodynamic curves obtained, as they are schematically given in figure 2 , have four characteristical domains:

1) an immunity domain (with hydrogen evolution),

2) a passivation domain: this potential area is more or less expanded. Its limits are, on one side, several peaks of weak current densities, characterizing equilibria iron/hydroxydes and hydroxydes and hydroxydes/oxydes (6-8), and on the other side, a potential Er (transpassivation potential above which pitting occurs),

3) a domain where pits grow,

4) a domain where the applied potential is decreasing: this determines potential Ep ("protection potential" or "end of pitting growth"). 


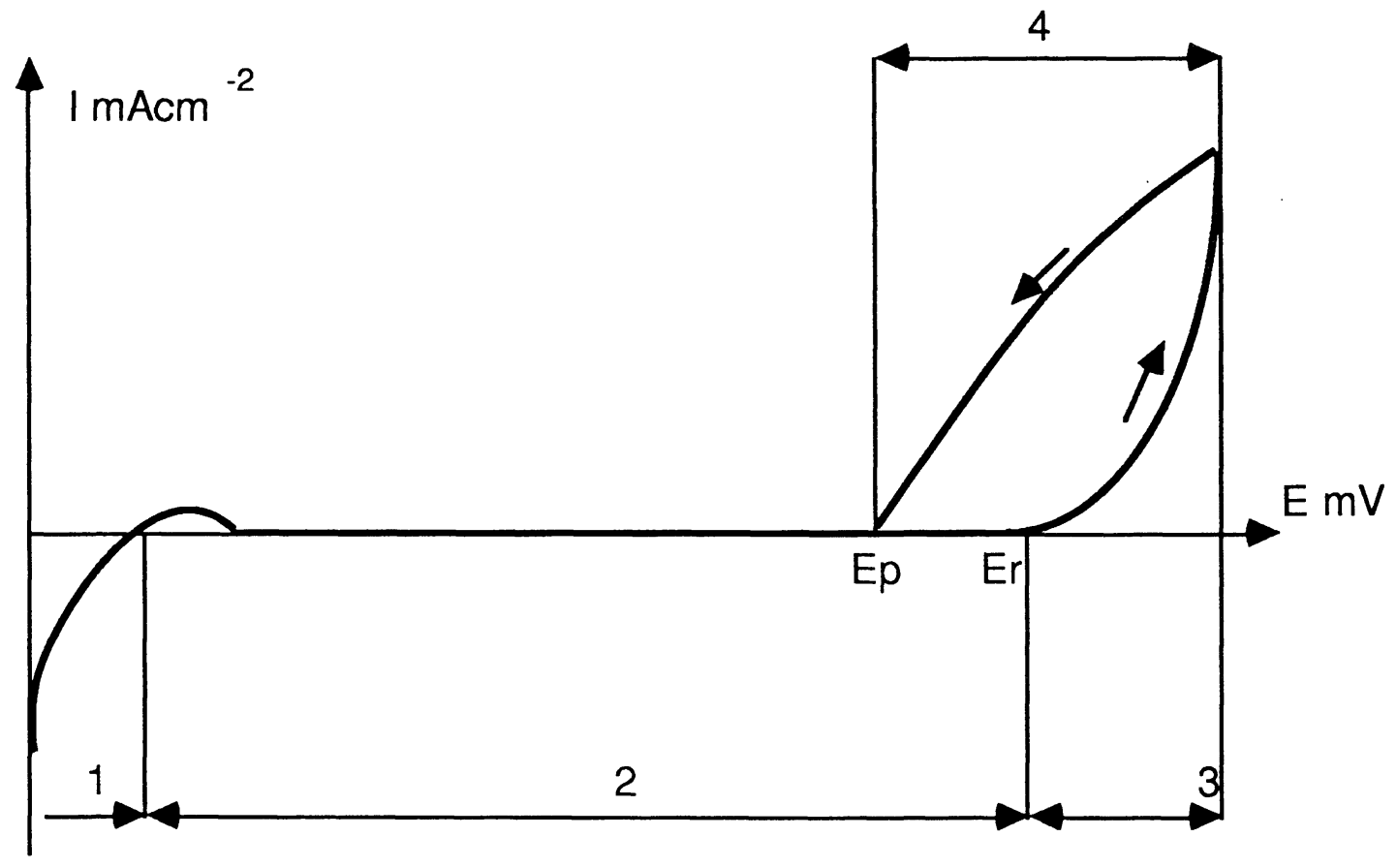

Fig. 2.-llustración esquemática de la curva potenciodinámica.

Fig. 2.-Schematic illustration of the potentiodynamic curve.

\section{RESULTADOS E INTERPRETACIÓN}

En cada serie de ensayos, trabajamos con tres concentraciones en iones cloruros, respectivamente $1,10^{-1}$ y $10^{-2} \mathrm{M}$.

En el estudio, la fuerza iónica no es tomada en cuenta.

\section{Influencia de la relación $\left[\mathrm{Cl}^{-}\right] /\left[\mathrm{OH}^{-}\right]$}

La primera serie de ensayos corresponde a disoluciones de sosa o de potasa. El dominio de $\mathrm{pH}$ estudiado varía entre 7 y 14 (Tabla 1).

La figura 3 muestra los potenciales Er y Ep en función del $\mathrm{pH}$ y del $\mathrm{pCl}=\operatorname{colog}\left[\mathrm{Cl}^{-}\right]$. En esta figura también se representa la línea recta de ecuación:

$$
\mathrm{pH}=12,5+0,0016 \mathrm{Er}
$$

definida por C. ANDRADE y S. GOÑI (4), para el dominio de $\mathrm{pH}$ incluido entre 12 y 13,6 y para concentraciones de cloruros de 0,5 y $0,75 \mathrm{M}$. Esta línea recta es compatible con nuestros resultados. Sin embargo, hay una pequeña diferencia por lo que se refiere a la influencia de los iones cloruro que puede explicarse por la mejor protección debida a la precipitación de productos cálcicos provenientes de las soluciones utilizadas por estos autores.

\section{RESULTS AND INTERPRETATION}

For each series of tests, three chloride contents were used, respectively $1,10^{-1}$ and $10^{-2} \mathrm{M}$.

In the whole investigation, the ionic forces of the solutions were neglected.

\section{Influence of the $\left[\mathrm{Cl}^{-}\right] /\left(\left[\mathrm{OH}^{-}\right]\right.$ratio}

This first series of tests dealt with $\mathrm{NaOH}$ and $\mathrm{KOH}$ solutions. The $\mathrm{pH}$ values studied were ranging between 7 and 14 (Table I).

Figure 3 gives potentials Er and Ep, versus the parameter $\mathrm{pCl}=$ colog $[\mathrm{Cr}]$. In this figure, the straight line representing the equation:

$$
p H=12,5+0,0016 E r
$$

is also drawn. It was defined by C. ANDRADE and S. GOÑI (4) for $\mathrm{pH}$ ranging between 12 and 13,6 and for chloride contents ranging between $0,5 \mathrm{M}$ and $0,75 \mathrm{M}$. This line is in agreement with our results. But it exists a difference concerning the influence of the chloride ions. This can be explained by the protection improvement due to the precipitation of calcium containing products in the solutions studied by these authors. 
TABLA | Table I

$\mathrm{NaOH}($ or $\mathrm{KOH})+\mathrm{NaCl}$

\begin{tabular}{|c|c|c|c|c|c|}
\hline $\mathrm{pH}(1)$ & $\mathrm{pCl}$ & Er $m V_{S C E}$ & Ep $\mathrm{mV}$ SCE & prod. (2) & $\mathrm{Ec} \mathrm{mV} V_{\mathrm{SCE}}$ \\
\hline 12,90 & 0 & -300 & -578 & $\mathrm{x}$ & \\
\hline 13,00 & 1 & +300 & -585 & $\mathrm{x}$ & -454 \\
\hline 12,84 & 0 & -350 & -600 & $\mathrm{x}$ & -577 \\
\hline 13,11 & 1 & +217 & -583 & $\mathrm{x}$ & -466 \\
\hline$\underline{11,90}$ & 0 & -450 & -650 & $\mathrm{x}$ & \\
\hline 11,95 & 1 & -300 & -639 & $\mathrm{x}$ & \\
\hline 11,90 & 2 & +110 & -617 & $\mathrm{x}$ & -573 \\
\hline 11,95 & 0 & -500 & -650 & $\mathrm{x}$ & $-587 *$ \\
\hline 11,95 & 1 & -350 & -646 & $\mathrm{x}$ & $-564 *$ \\
\hline 11,95 & 2 & -50 & -591 & $\mathrm{x}$ & $-494 *$ \\
\hline$\underline{10,60}$ & 0 & -480 & -662 & $\mathrm{M} / \mathrm{G}$ & \\
\hline 10,82 & 1 & -450 & -660 & $\mathrm{M} / \mathrm{G}$ & \\
\hline 10,82 & 2 & -350 & -659 & $\mathrm{M} / \mathrm{G}$ & \\
\hline 10,72 & 0 & -530 & -658 & $\mathrm{M} / \mathrm{G}$ & $-675 *$ \\
\hline 11,01 & 1 & -450 & -665 & $\mathrm{M} / \mathrm{G}$ & $-696 *$ \\
\hline 11,04 & 2 & -350 & -660 & $\mathrm{M} / \mathrm{G}$ & $-683 *$ \\
\hline 9,50 & 0 & -500 & -650 & $\mathrm{M} / \mathrm{G}$ & $-718 *$ \\
\hline 9,62 & 1 & -400 & -662 & $\mathrm{M} / \mathrm{G}$ & $-705^{*}$ \\
\hline 9,10 & 2 & -400 & -640 & $\mathrm{M} / \mathrm{G}$ & $-650 *$ \\
\hline 6,50 & 0 & -470 & -660 & $\mathrm{M} / \mathrm{G}$ & \\
\hline 6,50 & 1 & -400 & -650 & $\mathrm{M} / \mathrm{G}$ & \\
\hline 7,00 & 2 & -300 & -590 & $\mathrm{M} / \mathrm{G}$ & \\
\hline
\end{tabular}

(1) 12,90: $\mathrm{NaOH}$ 12,84: KOH. (2) x: óxido verde (Green Rust I). M: magnetita (magnetite). G: goetita (geothite). *: picaduras (pits).

Las curvas obtenidas concuerdan adecuadamente con el análisis de M. POURBAIX (9) sobre las circunstancias experimentales de corrosión en presencia de cloruros. En efecto, la figura 3 permite definir una zona de pasividad imperfecta, comprendida entre los potenciales de protección y de ruptura, y cuyo dominio de existencia varía según el $\mathrm{pH}$ considerado.

Cabe observar también que la naturaleza de los cationes $\left(\mathrm{Na}^{+}\right.$ó $\left.\mathrm{K}^{+}\right)$no parece tener influencia sobre los potenciales estudiados.

Las observaciones visuales sobre los productos de corrosión han dado los resultados siguientes:

- Para $\mathrm{pH} \leq 11$, la formación de picaduras es uniforme, y la magnetita precipita sobre la superficie del acero. La solución toma una coloración ocre debida a la presencia de partículas finas. La difracción de rayos $X$ muestra que se trata de goetita.
The curves obtained were in perfect agreement with the explanation given by $M$. POURBAIX (9) concerning the experimental conditions of corrosion in the presence of chlorides. Figure 3 indeed makes it possible to determine a domain of imperfect passivity, ranging between $E_{r}$ and $E_{p}$, this potential range depends on the solution $\mathrm{pH}$.

It is also to be noticed that the type of cations $\left(\mathrm{Na}^{+}\right.$or $\left.\mathrm{K}^{+}\right)$seems to have no effect on the critical potentials studied.

The visual examinations of corrosion products gave the following results:

- For $\mathrm{pH} \leq 11$, pitting occured uniformly on the whole steel surface and magnetite precipitated on this surface. The solution colour was yellow-brown due to the presence of fine particles. The $X$-ray diffraction showed out that it dealt with goethite. 


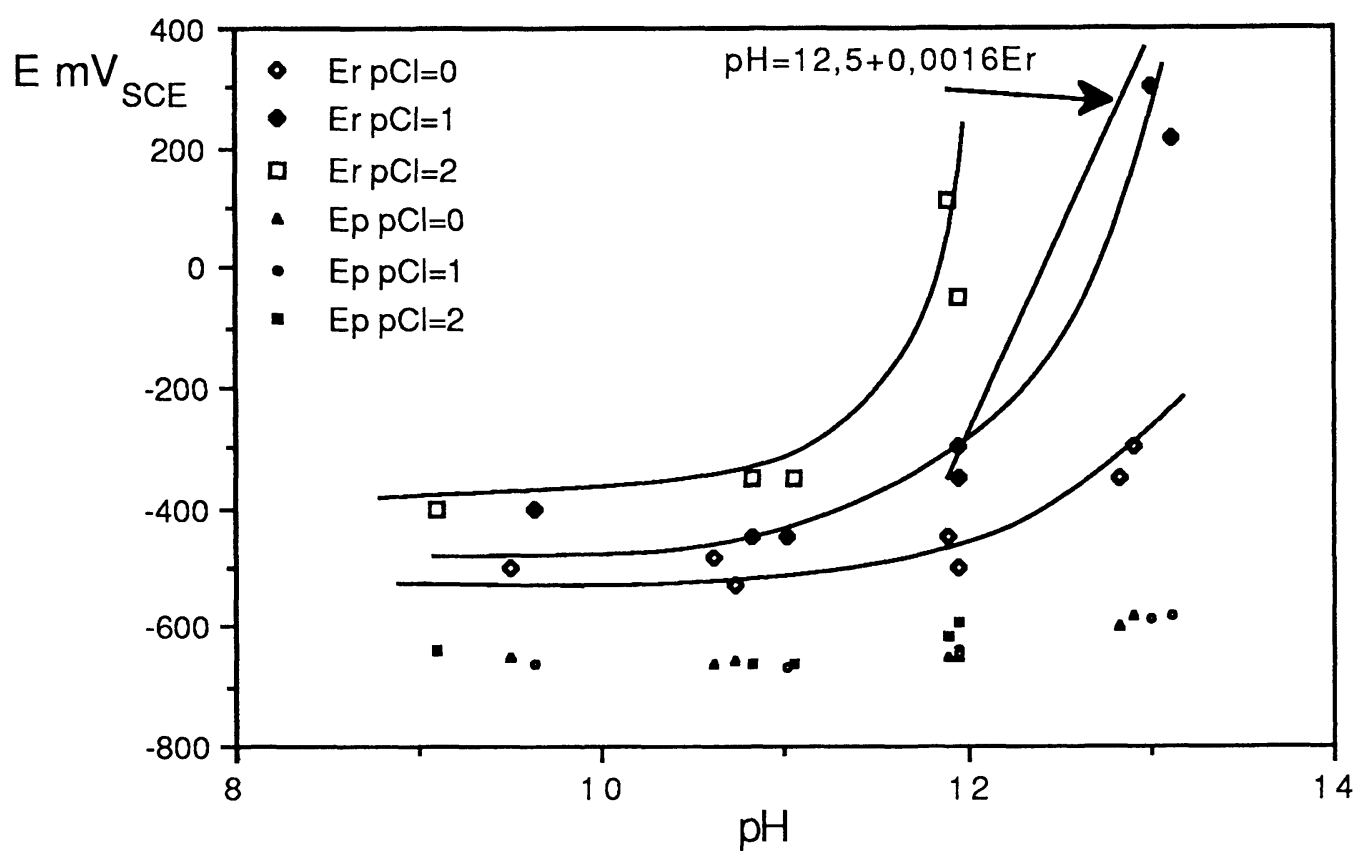

Fig. 3.- Influencia de los iones cluroro en los potenciales de picadura y de protección en soluciones de $\mathrm{NaOH}$ y $\mathrm{KOH}$.

Fig. 3.-Influence of chloride ions on pitting and protective potentials in $\mathrm{NaOH}$ or $\mathrm{KOH}$ solutions.

- Para $\mathrm{pH}>11$, aparece un producto verdoso cuya naturaleza es la misma del que se forma por oxidación de cloruro ferroso en medio básico. Este hidróxido clorurado, $\mathrm{Fe}_{4}(\mathrm{OH})_{8} \mathrm{Cl}$, llamado herrumbre verde I, se transforma en goetita por oxidación. Su presencia aisla el acero de la solución y conduce a una corrosión por picaduras intensa.

Finalmente el potencial de protección Ep nunca es inferior a $-700 \mathrm{mV}_{\text {SCE }}$ en todo el dominio estudiado.

\section{Influencia de los iones carbonatos}

Esta segunda serie de ensayos estudia disoluciones con varias concentraciones en carbonatos. En una primera etapa éstos fueron introducidos como $\mathrm{NaHCO}_{3}$ (Tabla II). La concentración no interviene en el valor del $\mathrm{pH}$, el cual permanece cercano a 8,5 (efecto tampón). En este caso la especie iónica preponderante en disolución es $\mathrm{HCO}_{3}^{-}$. Las variaciones del potencial $\mathrm{Er}$ observadas son indicadas en la figura 4 en función de $\mathrm{pHCO}_{3}=$ colog $\left[\mathrm{HCO}_{3}^{-}\right]$. El aspecto de las curvas obtenidas es similar al de las correspondientes a la influencia de los iones hidróxilos.

Puede observarse que existe también un umbral de concentración para la precipitación de los diferentes productos de corrosión: para valores superiores a $\mathrm{pHCO}_{3}=1$, los productos formados son la goetita y la magnetita. Para las fuertes concentraciones, aparece un compuesto floculento similar a la herrumbre verde I, pero de color blanco.
- For $\mathrm{pH}>11$, a flaky greenish product was formed, with an aspect similar to that of the product formed by oxyding ferrous chloride in an alkaline medium $(10,11)$. This hydroxyde which contains chlorine $\mathrm{Fe}_{4}(\mathrm{OH})_{8} \mathrm{Cl}$, is called Green Rust I. It changes into goethite after oxydation. When it is present, the steel surface is isolated from the solution, which results in a strong pitting.

At last, the protection potential Ep was never lower than - $700 \mathrm{mV}_{\text {SCE }}$, in the whole investigated domain.

\section{Influence of the carbonates ions}

This second series of tests dealt with solutions containing various carbonates contents. In a first stage, carbonates were introduced as $\mathrm{NaHCO}_{3}$ (Table II). The content has no effect on the $\mathrm{pH}$ value which remains about 8,5 (buffer effect). In this case, the most important ion is $\mathrm{HCO}_{3}^{-}$. The Er potential valued were plotted versus $\mathrm{pHCO}_{3}=\mathrm{colg}\left[\mathrm{HCO}_{3}^{-}\right]$. Figure 4 shows that the shape of the obtained curves is similar to that concernig the influence of hydroxyls ions.

A content limit also exists for the precipitation of the various corrosion products. It means that for $\mathrm{pHCO}_{3}>1$, the products formed are goethite and magnetite. For the highest contents, a flaky compound appears, similar to Green Rust l, but its colour is whitish. 
TABLA \| Table $\|$

$\mathrm{NaHCO}_{3}+\mathrm{NaCl}$

\begin{tabular}{|c|c|c|c|c|c|}
\hline $\mathrm{pHCO}_{3}$ & $\mathrm{pCl}$ & $\mathrm{Er} m \mathrm{~V}_{\mathrm{SCE}}$ & $\mathrm{Ep} \mathrm{m} \mathrm{V}_{\mathrm{SCE}}$ & prod. (1) & Ec mV $\mathrm{SCE}$ \\
\hline & & & & & \\
0,290 & 0 & -266 & -669 & $\mathrm{x}$ & $-546^{*}$ \\
0,290 & 0 & -350 & -479 & $\mathrm{x}$ & $-465^{*}$ \\
0,290 & 1 & +50 & -320 & $\mathrm{x}$ & $-189^{*}$ \\
0,534 & 2 & $?$ & $?$ & & -73 \\
0,534 & 0 & -308 & -550 & $\mathrm{x}$ & \\
0,534 & 0 & -317 & -510 & $\mathrm{x}$ & \\
0,534 & 1 & -50 & -450 & $\mathrm{x}$ & \\
1,290 & 2 & +520 & +200 & $\mathrm{x}$ & \\
1,290 & 0 & -534 & -600 & $\mathrm{M} / \mathrm{G}$ & $-574^{*}$ \\
1,290 & 1 & -534 & -667 & $\mathrm{M} / \mathrm{G}$ & $-748^{*}$ \\
1,290 & 2 & -517 & -625 & $\mathrm{M} / \mathrm{G}$ & $-743^{*}$ \\
1,534 & 0 & -100 & -480 & $\mathrm{M} / \mathrm{G}$ & $-758^{*}$ \\
1,534 & 1 & -583 & -621 & $\mathrm{M} / \mathrm{G}$ & \\
2,534 & 0 & -575 & -607 & $\mathrm{M} / \mathrm{G}$ & \\
2,534 & 1 & -600 & -656 & $\mathrm{M} / \mathrm{G}$ & $-660^{*}$ \\
2,534 & 2 & -383 & -661 & $\mathrm{M} / \mathrm{G}$ & \\
3,534 & 2 & -330 & -645 & $\mathrm{M} / \mathrm{G}$ & $-630^{*}$ \\
& & & & & \\
\hline
\end{tabular}

(1) x: producto blanquecino (whitish product). M: magnetita (magnetite). G: goetita (geothite). *: picaduras (pits).

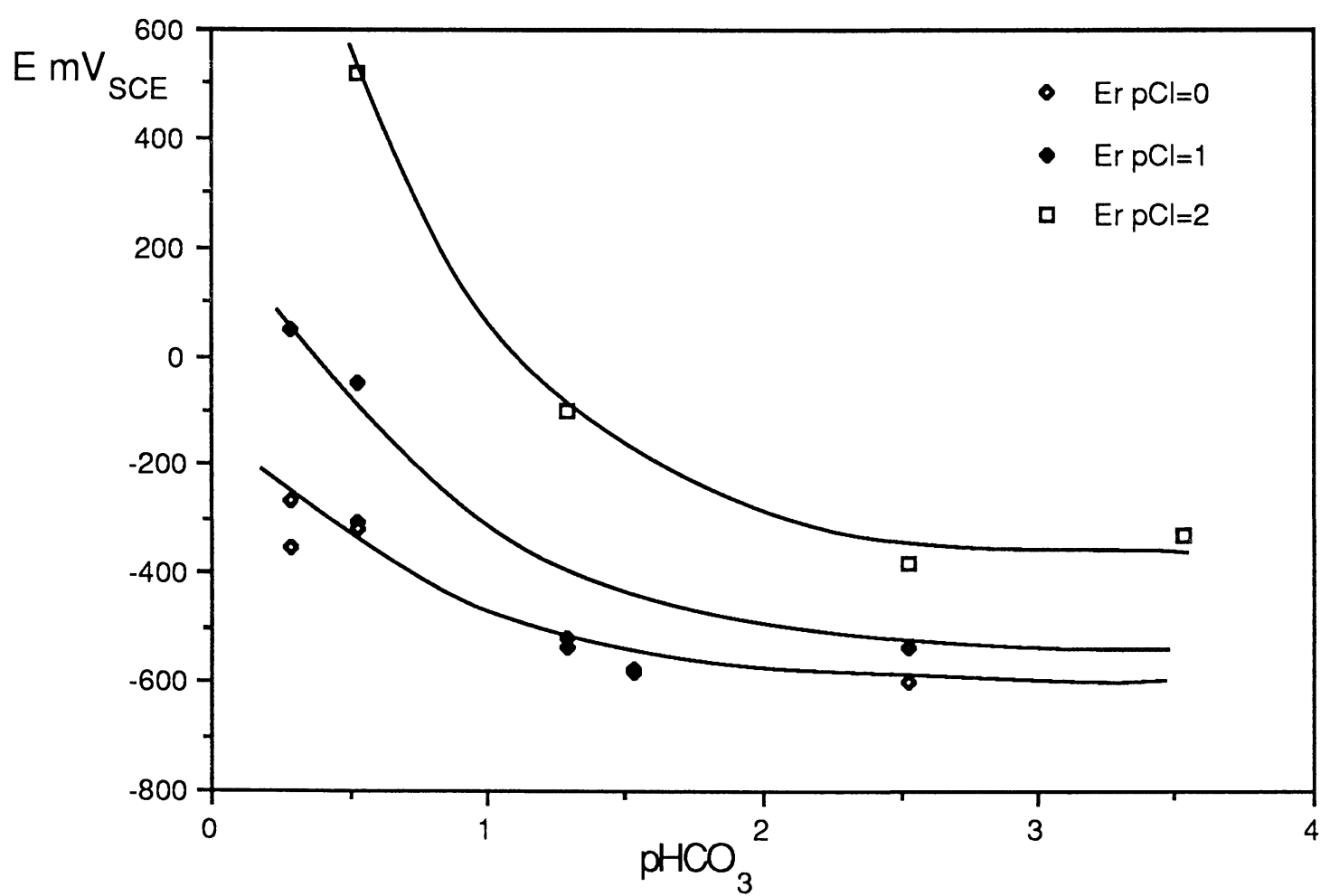

Fig. 4. - Influencia sobre el potencial de picadura de la presencia de iones cloruro en soluciones de $\mathrm{NaHCO}_{3}$.

Fig. 4.-Influence of chloride ions on pitting potentials in $\mathrm{NaHCO}_{3}$ solutions. 
TABLA III Table III

$\mathrm{Na}_{2} \mathrm{CO}_{3}+\mathrm{NaCl}$

\begin{tabular}{|c|c|c|c|c|c|}
\hline pAlk & $\mathrm{pCl}$ & Er mV SCE & Ep mV SCE & prod. (1) & Ec mV $V_{\text {SCE }}$ \\
\hline & & & & & \\
$-0,056$ & 0 & -50 & -595 & $\mathrm{x}$ & $-432^{*}$ \\
$-0,056$ & 1 & $?$ & $?$ & & -185 \\
$-0,056$ & 2 & $?$ & $?$ & & -171 \\
0,944 & 0 & -334 & -571 & $\mathrm{x}$ & \\
0,944 & 0 & -350 & -686 & $\mathrm{x}$ & $-560^{*}$ \\
0,944 & 0 & -373 & -541 & $\mathrm{x}$ & \\
0,944 & 1 & -160 & -588 & $\mathrm{x}$ & $-494^{*}$ \\
0,944 & 2 & +100 & -478 & $\mathrm{x}$ & $-401^{*}$ \\
1,944 & 0 & -450 & -652 & $\mathrm{x}$ & $-655^{*}$ \\
1,944 & 0 & -466 & -655 & $\mathrm{x}$ & \\
1,944 & 1 & -334 & -635 & $\mathrm{x}$ & $-637^{*}$ \\
1,944 & 1 & -410 & -635 & $\mathrm{x}$ & \\
1,944 & 2 & -110 & -584 & $\mathrm{x}$ & \\
1,944 & 2 & -167 & -570 & $\mathrm{x}$ & $-536^{*}$ \\
2,944 & 0 & -510() & -666 & $\mathrm{M} / \mathrm{G}$ & $-723^{*}$ \\
2,944 & 1 & -450 & -661 & $\mathrm{M} / \mathrm{G}$ & $-705^{*}$ \\
2,944 & 2 & -400 & -658 & $\mathrm{M} / \mathrm{G}$ & $-696^{*}$ \\
& & & & & \\
\hline
\end{tabular}

(1) X: producto blanquecino (whitish product). M: magnetita (magnetite). G: goetita (geothite). *: picaduras (pits).

Al igual que en las soluciones no carbonatadas, los potenciales de protección presentan un límite inferior a $-700 \mathrm{mV}_{\text {SCE }}$.

Los resultados obtenidos permiten precisar el efecto de los carbonatos sobre el comportamiento electroquímico de los aceros en diversas soluciones. Los iones carbonatos disminuyen el $\mathrm{pH}$ de las soluciones, pero no modifican las características químicas de las películas formadas bajo polarización (6-8). Por lo tanto, es conveniente tratar los carbonatos como los iones hidróxilos. Así, el parámetro a tomar en consideración no es la relación $\left[\mathrm{Cl}^{-}\right] /\left[\mathrm{OH}^{-}\right]$, sino la relación $[\mathrm{Cr}] /[\mathrm{AIK}]$, en la cual AlK representa la suma de las bases titrables en equivalentes por litro. Esta hipótesis es utilizada en las figuras 5 y 6 , que muestran:

- los resultados obtenidos con los iones bicarbonatos e hidróxilos, y,

- una nueva serie de valores de los potenciales críticos obtenida del estudio de disoluciones de carbonato de sodio, $\mathrm{Na}_{2} \mathrm{CO}_{3}$ (Tabla III). Este compuesto permite definir soluciones tampón en las cuales las concentraciones en iones $\mathrm{HCO}_{3}^{-}$y $\mathrm{CO}_{3}{ }^{2-}$ son iguales. En este caso, la alcalinidad total se escribe:

$$
[\mathrm{AIK}]=\left[\mathrm{OH}^{-}\right]+\left[\mathrm{HCO}_{3}{ }^{-}\right]+2\left[\mathrm{CO}_{3}{ }^{2-}\right]
$$

As for the non carbonated solutions, the protection potential Ep had a lower limit equal to $-700 m V_{S C E}$.

The influences of carbonates on the electrochemical behaviour of steel in the various solutions can be described by the results obtained, as follows. The carbonates ions decreases the solution $\mathrm{pH}$, but they do not change the chemical properties of the films formed under polarization $(6,8)$. So, it is convenient to treat carbonates like hydroxyls ions. The parameter to be taken into account is therefore, not the $[\mathrm{Cr}] /[\mathrm{OH}]$ ratio, but preferably the [Cr]/[AIK] ratio, where AIK is the total of the contents (in equivalent per liter) of the alkaline species. This assumption was used in figures 5 and 6 which show:

- the results obtained with bicarbonates and hydroxyls ions and,

- another series of values of the critical potentials, resulting from the investigation on $\mathrm{Na}_{2} \mathrm{CO}_{3}$ (Table III). This compound makes it possible to define buffer solutions, at $\mathrm{pH}=$ 10,3 , for which the $\mathrm{HCO}_{3}^{-}$and $\mathrm{CO}_{3}{ }^{2-}$ contents are equal. In this case the total alkalinity is:

$$
[\mathrm{AIK}]=[\mathrm{OH}]+\left[\mathrm{HCO}_{3}^{-}\right]+2\left[\mathrm{CO}_{3}^{2-}\right]
$$



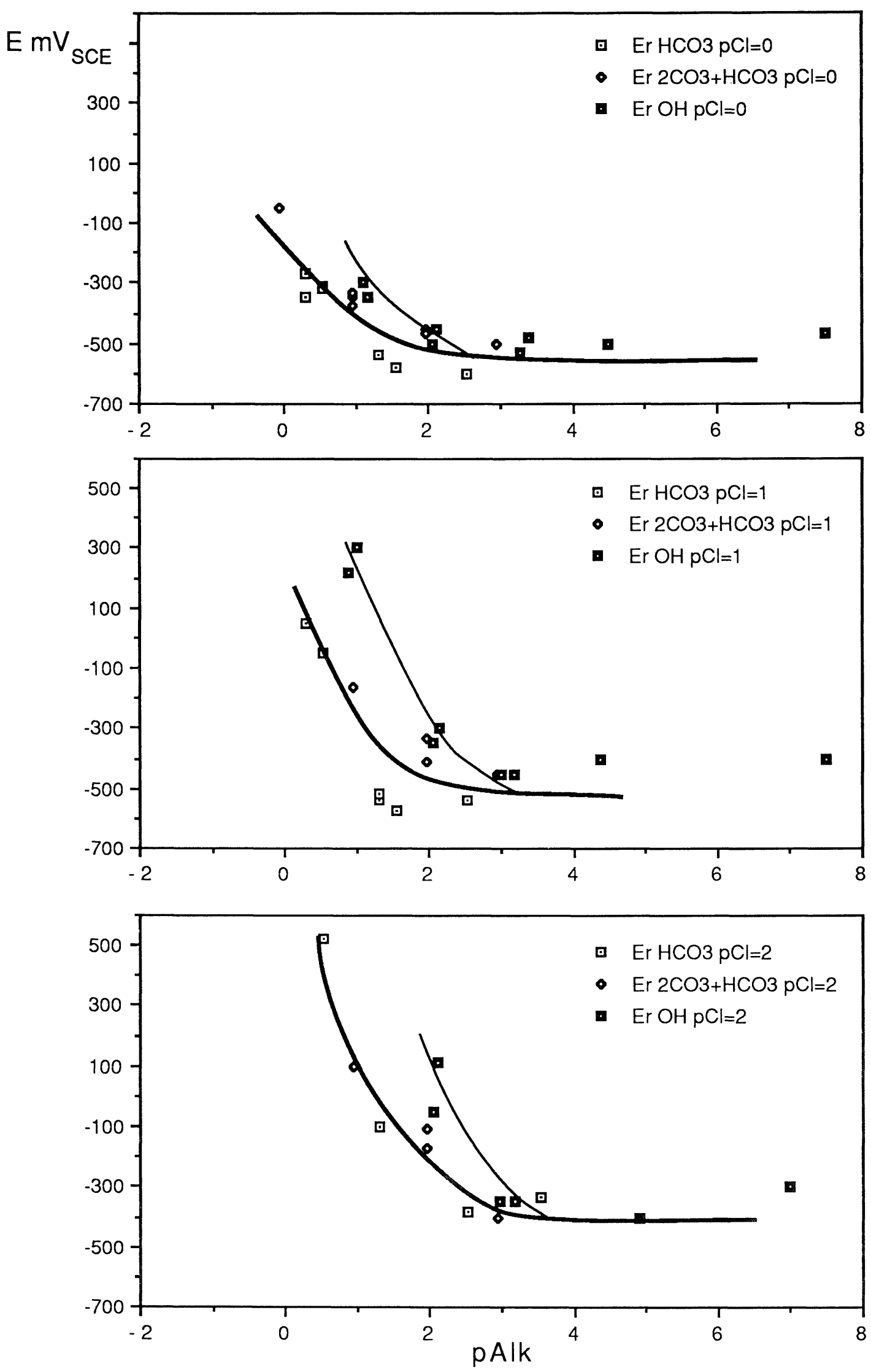

Fig. 5. - Variación de los potenciales de picadura en función de pAlc. Influencia de los valores de pCl.

Fig. 5.-Variation of pitting potentials in function of $p A l k$. Influence of $p C l$ values. 

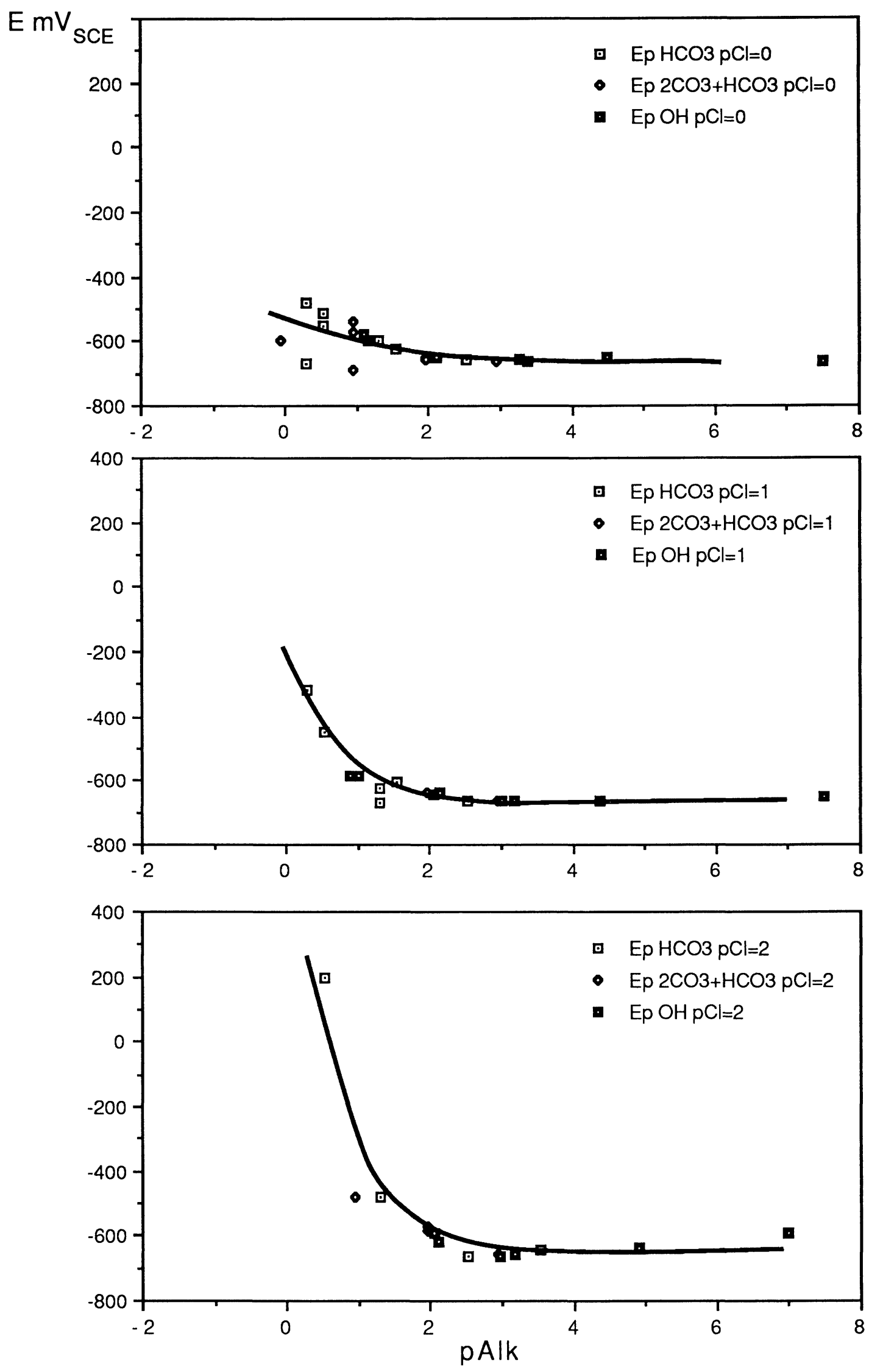

Fig. 6.-Variación de los potenciales de protección en función de pAlc. Influencia de los valores de pCl.

Fig. 6. - Variation of protective potentials in function of $p A l k$. Influence of $p C l$ values. 

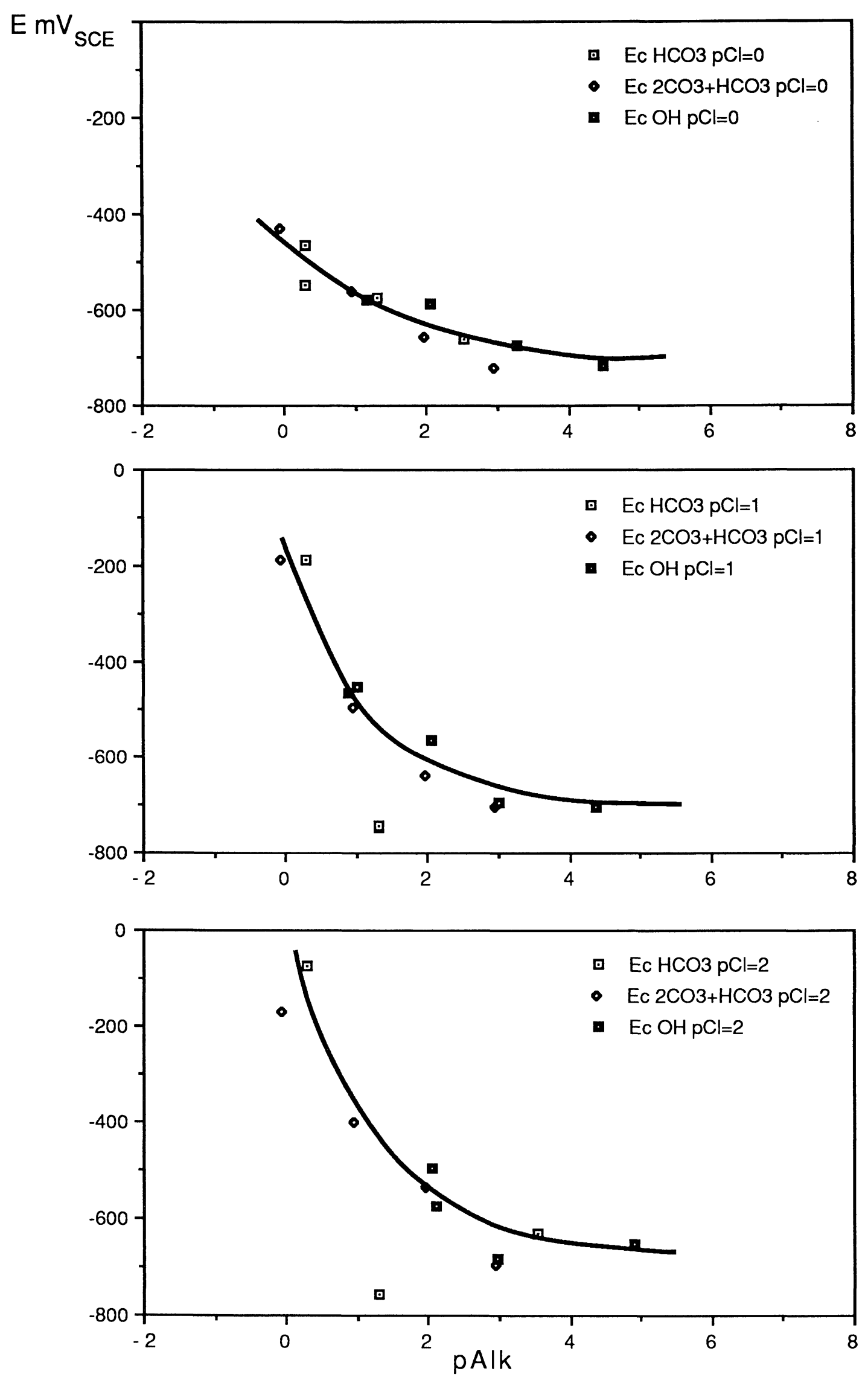

Fig. 7.-Variación de los potenciales de corrosión en función de pAlc. Influencia de los valores de pCl.

Fig. 7.-Variation of corrosion potentials in function of pAlk. Influence of $p C l$ values. 
La variación del potencial de protección Ep (figura 6) en función de pAlK es entonces representada, a $\mathrm{pH}$ constante, por una curva única. Ocurre lo mismo con el potencial de ruptura Er (figura 5) para soluciones menos básicas. Las divergencias en las altas alcalinidades se pueden explicar teniendo en cuenta los siguientes factores:

- la calidad de la película de pasivación (porosidad) puede favorecer la difusión de los iones cloruros,

- y además, la alcalinidad a considerar no es la alcalinidad total de la solución inicial, sino la que corresponde a la zona próxima al acero. En efecto, la mobilidad eléctrica del ion hidróxilo siendo cerca de tres veces superior a la de los otros iones, la naturaleza de la solución en contacto con el metal es totalmente diferente de la del resto de la solución.

\section{Potenciales de abandono}

La figura 7 muestra que el potencial de abandono Ec es también función de $[$ AlK $]=\left[\mathrm{OH}^{-}\right]+$ $+\left[\mathrm{HCO}_{3}{ }^{-}\right]+2\left[\mathrm{CO}_{3}{ }^{2-}\right]$. Sin embargo, es importante señalar que cuando el medio contiene carbonatos, las picaduras aparecen para cualquier nivel de la alcalinidad total, mientras que el acero está protegido cuando la concentración en iones hidróxilo es superior a $10^{-2} \mathrm{M}$. Esta observación confirma la regla de HAUSMANN (12) según la cual el acero se corroe a una velocidad notable si la concentración en cloruros sobrepasa un umbral que depende únicamente del $\mathrm{pH}$. Otros estudios (13) ya han verificado esta regla.

\section{Aplicaciones a la protección catódica}

La protección catódica ha llegado a ser un método corriente de protección contra la corrosión en diversas industrias. Muchos datos (15) han sido publicados acerca del uso de esta técnica para proteger los tableros o lozas de hormigón armado. Además de las condiciones técnicas para su instalación en la obra, un aspecto fundamental es la elección de un criterio optimal de protección.

En la figura 8 están indicadas las diferencias entre el potencial de corrosión y el potencial de protección para cada disolución estudiada. La distribución de los puntos muestra que puede ser arriesgado utilizar la regla que consiste en polarizar el acero desplazando el potencial de corrosión de un valor fijo. En efecto, una polarización de este tipo puede conducir, para las fuertes alcalinidades, a una subprotección, y para los otros medios, a una sobreprotección, no justificada.
The protection potential Ep change versus $p A I K$ is given by a unique curve (figure 6), for constant $\mathrm{pCl}$. The transpassivation potential Er gave the same result (figure 5) when the solution $\mathrm{pH}$ was not too high. The discrepancies for the highest $\mathrm{pH}$ values can be explained with the two following factors:

- on one hand, the quality (porosity, etc.) of the passivation film can enhance the chloride diffusion,

- on the other hand, the alkalinity to be taken into account is not the value concerning the initial solution but that wich corresponds to the solution close to the steel surface. The electrical mobility of the dydroxyl ion is indeed three times higher than that of any other ion, so the solution close to the metal is quite different from the bulk solution.

\section{Zero-current potentials}

Figure 7 shows that the zero-current potential Ec is also depending on the parameter $[\mathrm{AIK}]=[\mathrm{OH}]+\left[\mathrm{HCO}_{3}^{-}\right]+2\left[\mathrm{CO}_{3}{ }^{2-}\right]$. Nevertheless, it is of importance to notice that when carbonates are present in the solution, pitting occurs whatever the total alkalinity, as steel is protected when the $\mathrm{OH}$ content is higher than $10^{-2} \mathrm{M}$. This result confirms the HAUSMANN rule (12) according to which the corrosion rate is significant if the chloride content is higher than a threshold which depends only on the $\mathrm{pH}$. Other investigations (13) have already confirmed this rule.

\section{Application to cathodic protection}

Cathodic protection has become an usual protection method in various industries. Many data (14) have been published concerning the possibility of applying this technique for the protection of reinforced concrete bridge decks. The basic aspects to be considered are the technical conditions of operating, but also the choice of the protection criterion.

Figure 8 shows the differences between Ec (zerocurrent potential) and Ep (protection potential), for every studied solution. The scattering of the obtained points shows that it is dangerous to use a rule which would consist of only shifting the steel potential with a constant value. Such a polarization can indeed lead, for high $\mathrm{pH}$ solutions, to an unsufficient protection as for the other solutions, it would result in a useless overprotection. 


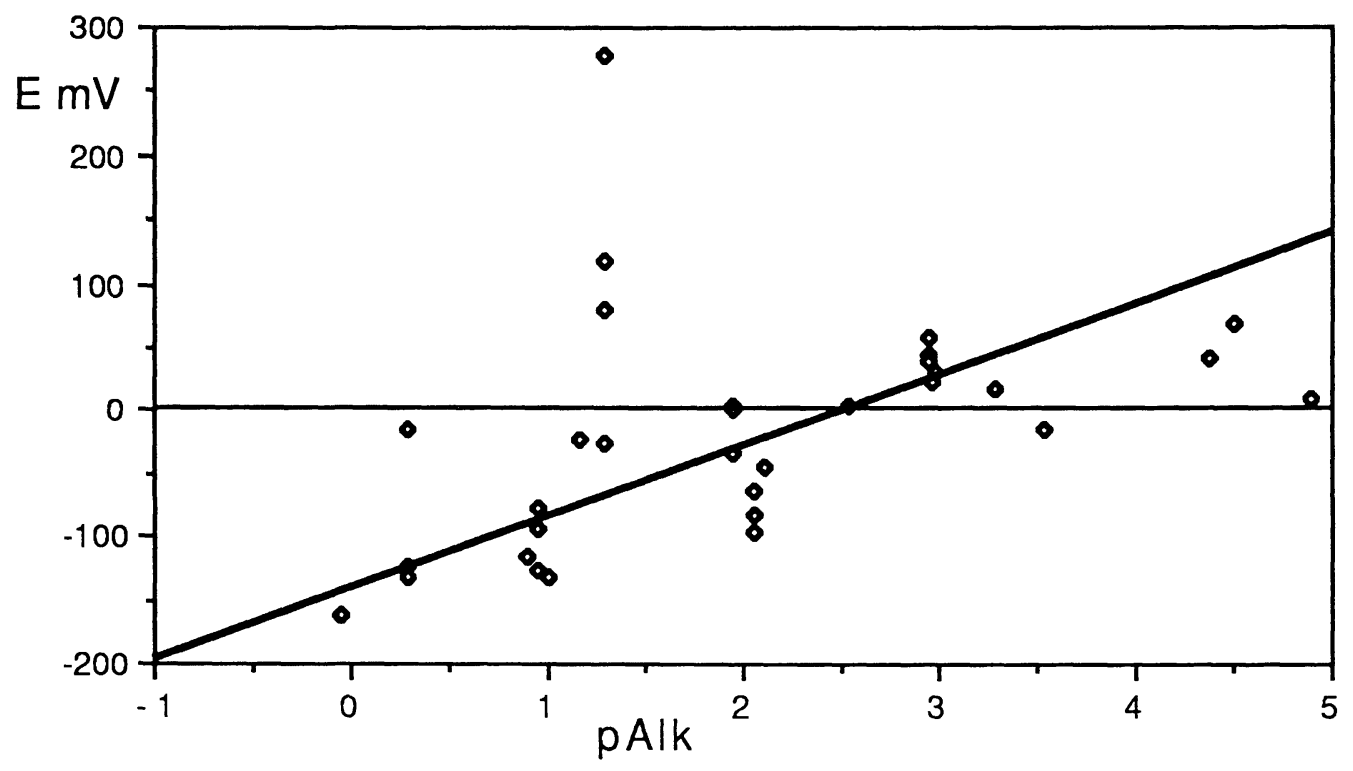

Fig. 8.-Ep-Ec en función pAlc (todas las soluciones ensayadas).

Fig. 8. - Ep-Ec versus pAlc (all tested solutions).

Por lo tanto, es preferible utilizar un potencial de polarización catódica bien determinado que conduzca a la inmunidad del metal. Sin embargo, para evitar el desprendimiento de hidrógeno que puede producirse en este caso, hay que limitarse a una polarización que conduzca a una pasividad perfecta de los aceros.

Se desprende de nuestros resultados que el límite superior del potencial a aplicar se situa a - $700 \mathrm{mV}_{\text {SCE }}$. Este criterio conviene al conjunto de las disoluciones estudiadas y a fortiori al medio constituido por el hormigón, para el cual debe utilizarse un coeficiente de seguridad para prevenir efectos locales de corrosión.

\section{CONCLUSIÓN}

Este estudio fue emprendido con la finalidad de determinar la influencia de la fase líquida de los poros del hormigón sobre el comportamiento electroquímico de las armaduras de acero a diversos potenciales. Las disoluciones sintéticas utilizadas, altamente básicas, son o no carbonatadas y cubren playas de concentración importantes.

Los parámetros estudiados son el potencial de ruptura Er, de protección Ep y de abandono Ec. El estado de los aceros fue examinado visualmente y ciertos productos de corrosión fueron analizados por difracción de rayos $X$. Las principales conclusiones que pueden formularse son las siguientes:
So, the protection criterion to be used would be preferably the cathodic potential which has a value resulting in the metal immunity. However, the hydrogen evolution at such potentials are to be avoided, so the polarization must be limited to potentials resulting to a perfect passivity of steel.

The results obtained show out that the potential to be applied is less than - $700 \mathrm{mV}$ SCE. This criterion is convenient for every solution tested and therefore to concrete, where a safety coefficient must be used for preventing any local corrosion condition.

\section{CONCLUSIONS}

The object of the present investigation was to study the influence of the pore solution in concrete on the electrochemical behaviour of embedded steel, at various potentials. The synthetical solutions used were of high alkalinity, and carbonated or not. Their anions contents were ranging in large intervals.

The studied parameters are the transpassivation potential Er, the protection (agains pitting corrosion) Ep and the zero-current potential Ec. The state of the steel surface was wisually examined and some corrosion products were analyzed by $X$-ray diffraction. The main conclusions to be drawn are as follows: 
1) La relación $\left[\mathrm{Cl}^{-}\right] /\left(\left[\mathrm{OH}^{-}\right]+\left[\mathrm{HCO}_{3}^{-}\right]+2\left[\mathrm{CO}_{3}{ }^{2-}\right]\right)$ influye sobre las propiedades electroquímicas del acero polarizado o no. La relación $\left[\mathrm{Cl}^{-}\right] /\left[\mathrm{OH}^{-}\right]$debe ser utilizada únicamente para la determinación de la existencia de una corrosión por picaduras en la ausencia de polarización.

2) Los productos particulares, que precipitan sobre el acero, son la herrumbre verde I y sus "derivados carbonatados". Estos productos obtenidos fácilmente por síntesis son rara vez señalados en las obras de hormigón armado puesto que se transforman rápidamente en productos (óxidos, etc) más estables al contacto con la atmósfera. Su presencia dentro del hormigón conduce a una corrosión por picaduras intensa aislando el acero del medio que le rodea.

3) El mejor criterio de protección catódica consiste en imponer un potencial de polarización bien definido. El valor de dicho umbral se situa a $-700 \mathrm{mV}_{\text {SCE }}$ o, para una mejor seguridad, a valores más negativos.
1) The electrochemical behaviour of steel, which is under or without polarization, is influenced by the parameter $[\mathrm{Cr}] /\left([\mathrm{OH}]+\left[\mathrm{HCO}_{3}^{-}\right]+\right.$ $\left.+2\left[\mathrm{CO}_{3}{ }^{2-}\right]\right)$. The $[\mathrm{Cr}] /\left[\mathrm{OH}^{-}\right]$ratio is to be used only for determining the existence of pitting in the absence of applied potential.

2) The particular products formed on the steel surface are Green rust-I and its "carbonated derivatives". These compounds are easily obtained by synthesis, but they are rarely reported for real cases because they are quickly changed into products (oxides, etc) which are more stable in contact of the atmosphere. When they are present in concrete, a significant pitting likely occurs because the steel is isolated from its environment (concrete).

3) The best criterion to be used for cathodic protection is the value of the applied potential. The threshold value is - $700 \mathrm{mV}$ SCE , or, for a better safety, lower potentials.

\section{REFERENCIAS}

\section{REFERENCES}

(1) J. K. BOAH, S. K. SOMUAH and P. LEBLANC: Corrosion NACE 46, 2, pp. 153-158 (1990).

(2) A. MACIAS and C. ANDRADE: Corrosion Science, 30, 4/5, pp. 393-407 (1990).

(3) G. I. OGUNDELE: Corrosion NACE, 45, 12 pp. $981-984$ (1989).

(4) S. GOÑI and C. ANDRADE: Cement and Concrete Research, 20, pp. 525-539 (1990).

(5) M. B. IVES, Y. C. LU and J. L. LUO: Corrosion Science, 32, 1, pp. 91-102 (1991).

(6) J. FLIS, H. ORANOWSKA and Z. SZKLARSKA-SMIALOWSKA: Corrosion Sicience, 30, 11, pp. 1.085-1.099 (1990).

(7) E. B. CASTRO, J. R. VILCHE and A. J. ARVIA: Corrosion Science, 32, 1, pp. $37-50$ (1991).

(8) A. RAHARINAIVO, J. P. GUILBAUD, G. CHAHBAZIAN and F. DERRIEN: Corrosion Science (to be published 1992).

(9) M. POURBAIX: Leçons en corrosion électrochimique, pp. 262-263, 2ème Ed. CEBELCOR (1975).

(10) J. M. GENIN, A. BERAL and D. R. REZEL: Rapport Technique 297, CEBELCOR, 157, pp. $349-362$ (Nov. 1989).

(11) R. DRON: Private communication (1989).

(12) D. A. HAUSMANN: Materials Protection, pp. 19-22 (1967).

(13) A. RAHARINAIVO and J. M. GENIN: Materiales de Construcción, 36, 204, pp. 5-16 (1986).

(14) K. KENDELL and D. A. LEWIS: Contractor Report 4, Transport and Road Research Lab. (1984). 